\title{
LA COMPRENSIÓN DE TEXTOS DESDE UN ENFOQUE MULTICOMPONENCIAL. EL TEST "LEER PARA COMPRENDER”
}

\section{TEXT COMPREHENSION FROM A MULTICOMPONENTIAL PERSPECTIVE. THE “LEER PARA COMPRENDER” TEST}

\author{
Valeria Abusamra \\ Romina Cartoceti \\ Aldo Ferreres \\ Universidad de Buenos Aires, Argentina \\ Rossana De Beni \\ Cesare Cornoldi \\ Università degli Studi di Padova, Italia
}

\begin{abstract}
Resumen: La comprensión de textos es una tarea compleja que requiere de la participación de múltiples habilidades cognitivas. Fallas en una o varias de estas habilidades pueden originar dificultades en la comprensión del nivel textual. La evaluación de dichas dificultades requiere, por lo tanto, una perspectiva multicomponencial. El incipiente desarrollo de la dimensión textual en la educación y en la clínica de las dificultades del aprendizaje plantea la necesidad de contar con un instrumento de esta naturaleza en la población hispanohablante. Con este objetivo se realizó la adaptación al español de un test (Nuova guida alla comprensione del testo, De Beni et al., 2003) para evaluar la comprensión de textos y se obtuvieron datos normativos para niños de 9 a 12 años. El test Leer para Comprender (Abusamra et al., en prensa) toma como base un modelo global de once componentes: esquema básico del texto, hechos y secuencias, semántica léxica, estructura sintáctica, cohesión textual, inferencias, intuición del texto, jerarquía del texto, modelos mentales, flexibilidad y errores e incongruencias. Este instrumento resulta sensible a la especificidad de los procesos implicados en la comprensión de texto. Un acercamiento desde una perspectiva multicomponencial permite no sólo operar de modo específico sobre los distintos aspectos de la comprensión sino que resulta, además, de gran utilidad para intervenir de manera focal sobre los procesos o habilidades implicados.
\end{abstract}

Palabras clave: comprensión lectora, evaluación, modelo multicomponencial.

\begin{abstract}
Text comprehension is a complex task that requires and involves multiple cognitive abilities. Deficits in one or several abilities can cause difficulties in understanding the textual level. Evaluating these impairments therefore requires a multicomponential perspective. The incipient development of the textual dimension of the learning difficulties in education as well as in clinic, elicits the need for an instrument of this nature for Spanish-speaking populations. With this objective, a Spanish version of a test (/Nuova guida alla comprensione del testo/, De Beni et al., 2003) was designed for evaluating text comprehension. Normative data for children of 9 to 12 years of age were obtained. The Leer para Comprender Test (Abusamra et al., in press) is based on a global model composed by eleven factors: basic text scheme, facts and sequences, lexical semantics, syntactic structure, textual cohesion, inferences, text sensibility, text hierarchy, mental models, flexibility, and errors and inconsistencies. This instrument is sensitive to the specificity of the processes implied in text comprehension. An approach from a multicomponential perspective not only allows operating specifically with the different aspects of comprehension, but it is also advantageous to intervene in a focused manner in the implied processes and abilities.
\end{abstract}

Keywords: reading comprehension, evaluation, multicomponential model.

\section{INTRODUCCIÓN}

Cada vez que escuchamos o leemos un mensaje verbal, ponemos en juego mucho más que nuestra capacidad de procesar palabras y oraciones aisladas.
Si bien la comprensión exitosa requiere de un lector u oyente que identifique palabras, que reconozca estructuras sintácticas y que pueda extraer significado de las oraciones individuales, comprender palabras u oraciones no completa la totalidad del proceso. 
La identificación de relaciones entre las distintas partes del texto y el conocimiento de mundo del lector constituyen un punto crucial en el proceso de comprensión. En consecuencia, el texto es percibido y representado en la memoria como una estructura coordinada y coherente más que como un conjunto desarticulado de piezas de información.

En este sentido, el proceso de comprensión ha sido comparado con el armado de un rompecabezas. Armar un rompecabezas implica juntar pequeños fragmentos en función de formar un todo unificado. El resultado de este mecanismo de integración es una representación interrelacionada en la cual las partes dejan ya de tener sentido por sí mismas. La metáfora del rompecabezas permite entender cómo se accede al sentido final de un texto: la construcción de una representación mental coherente de los hechos descriptos supone la integración de elementos individuales.

La comprensión de textos implica, entonces, un proceso complejo y activo en el que intervienen factores lingüísticos, psicolingüísticos, culturales y factores relacionados con la experiencia personal del sujeto.

Hasta los años '80, la mayoría de los trabajos abocados al estudio de la dimensión textual consideraban que el fenómeno de la comprensión debía ser analizado focalizando sobre los elementos estructurales que componían el texto. Es decir, el interés estaba puesto en llevar a cabo una descripción estrictamente lingüística. Sin embargo, hay claras evidencias de que el texto no implica solo lenguaje en sí mismo y menos aun una simple secuencia de oraciones aisladas. Comprender lo que leemos implica entender la relación entre lo que nos quieren decir y lo que de hecho nos dicen, entre lo dicho y lo no dicho; entre lo explícito y lo implícito. Un texto no dice todo; es un producto incompleto que requiere de un lector que reponga constantemente la información faltante. Umberto Eco (1997) destaca "todo texto es una máquina perezosa que le pide al lector que le haga parte de su trabajo. Pobre del texto si dijera todo lo que su destinatario debiera entender: no acabaría nunca".

En los últimos treinta años, la expansión de la Psicología cognitiva y la inclusión de la pragmática en los estudios (neuro)psicolingüísticos llevaron a la elaboración de nuevos marcos teóricos y modelos del nivel textual. Particularmente, la publicación de Mental models de
Johnson-Laird (1983) y Strategies in discourse comprehension de van Dijk y Kintsch (1983) establecieron una ruptura en la perspectiva dominante hasta el momento. El foco se desplaza hacia las representaciones mentales de las situaciones verbalmente descriptas y se postulan lo que se conoce como modelos mentales (Johnson-Laird, 1983) o de situación (van Dijk \& Kintsch, 1983, Kintsch, 1988). Estos modelos asumen que los lectores, cuando comprenden, construyen representaciones mentales del mundo real o ficticio al que el texto se refiere. Los modelos de situación son representaciones mentales de los personajes, los objetos, los escenarios, los eventos y las acciones descriptos en un texto. El lector compone un micromundo mental en base a la interacción entre el texto explícito y el conocimiento que tiene del mundo. Entonces, como dijimos antes, el proceso de comprensión comienza a ser visto como una actividad dinámica y cognitivamente compleja en la que el "comprendedor" construye una representación mental del texto.

Desde esta perspectiva, quien comprende debe actualizar continuamente sus representaciones mentales. Los personajes pueden desplazarse hacia nuevos escenarios, hay objetos que pueden quedar atrás, eventos que ya no son relevantes y sin alguna forma de actualización, se hace difícil una comprensión exitosa.

Este cambio de perspectiva en el estudio de la comprensión tuvo una fuerte incidencia en la redefinición del rol del lenguaje. El lenguaje ya no es pensado estrictamente en términos de información a ser analizada sintáctica y semánticamente para luego almacenarse en la memoria, sino que se lo ve como un conjunto de instrucciones de procesamiento acerca de cómo construir una representación mental del contenido del texto (Gernsbacher, 1990).

El interés renovado por las habilidades de comprensión lectora tuvo implicancias en la promoción de las competencias que intervienen en el aprendizaje normal o en el de los alumnos con dificultades específicas de comprensión. Es importante advertir que la diversidad del proceso en juego promueve que diferentes perspectivas y procedimientos diagnósticos puedan llegar a categorizaciones diferentes de las habilidades de comprensión.

Por ejemplo, diferentes condiciones en la evaluación de la comprensión de textos - con o sin texto presente, preguntas abiertas o elección de respuestas múltiples - relativizarán los 
resultados y pondrán de manifiesto elementos de especificidad.

Nuestro objetivo fue abordar los procesos de comprensión lectora desde un modelo de múltiples componentes. Comprender un texto no constituye una habilidad unitaria sino que integra competencias independientes unas de otras. En este marco, una aproximación multicomponencial a los procesos que median la dimensión textual es esencial tanto desde un punto de vista teórico como empírico.

Nos propusimos (1) adaptar al español un test (Nuova Guida alla Comprensione del Testo, De Beni et al., 2003) para evaluar la comprensión de textos que toma como base un modelo de componentes básicos del nivel textual, (2) obtener datos normativos para una población de niños de entre 9 y 12 años y (3) analizar el efecto de dos variables relativas al sujeto: grado y nivel de oportunidades educativas.

El Test Leer para comprender se basa en un modelo multicomponencial de lectura que integra once áreas (ver Figura 1). La noción de que dentro de la comprensión del texto pueden ser identificados subcomponentes específicos de dominio ya fue planteada en 1944 en un trabajo de Davis.

El autor mostró que la comprensión no es una capacidad o una operación mental unitaria sino que están involucradas habilidades mentales específicas relacionadas con factores diversos, independiente unos de otros (Davis, 1972).

Esta idea ha encontrado sustento a nivel teórico y empírico ya que no sólo se ha alcanzado mayor adecuación explicativa en la fundamentación de los procesos implicados sino que además, la evaluación de los alumnos con dificultad y la intervención desde una aproximación multicomponencial ha traído ventajas indiscutibles.

El modelo que sirvió de base para el diseño del test (De Beni et al., 2003) propone que el lector, cuando se enfrenta a un texto, debe ser capaz de reconocer, en primer lugar, los personajes, el lugar, el tiempo y los hechos presentes.

Esta habilidad, que evalúa la estructura típica de un texto, se conoce como Esquema básico del texto.

En segundo lugar, la comprensión de un texto - particularmente los narrativos, expositivos y científicos - depende de la capacidad del lector de individualizar los hechos y las secuencias propias de cada tipo textual. De esta manera, es importante que el lector pueda distinguir los hechos que se relacionan con las diferentes tipologías textuales e identificar la cronología fáctica desarrollada en la historia en términos de identificación de Hechos y secuencias.

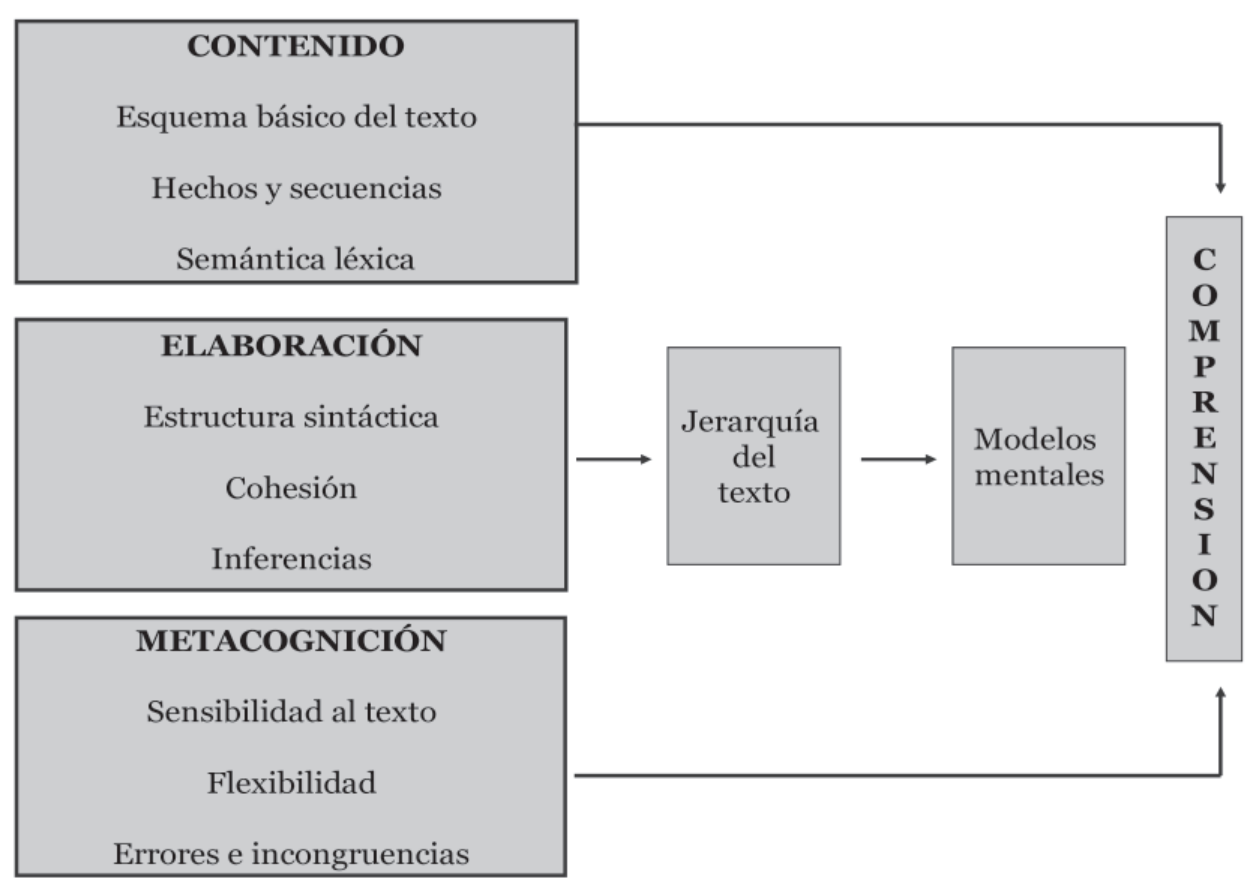

Figura 1. Esquema descritptivo de la relación entre las áreas implicadas en la comprensión de textos 
Otro aspecto esencial tiene que ver con la capacidad de establecer una red de relaciones entre las palabras que lo conforman. El lector debe, entonces poseer ciertas habilidades psicolingüísticas fundamentales que le permitan identificar relaciones de significado locales como la sinonimia, la paronimia y la antonimia y relaciones entre las palabras y oraciones del texto (Semántica léxica).

A partir del significado de las palabras, el lector puede acceder a la correcta elaboración de nexos gramaticales y sintácticos no sólo dentro del marco oracional sino también entre oraciones. En este sentido es que hablamos de capacidad de procesar la Estructura sintáctica de un texto. Los textos no ofrecen de manera explícita toda la información sino que requieren de un comprendedor activo que pueda establecer relaciones entre los elementos textuales y las ideas contenidas en ellos. Conectores, anáforas y otros elementos de cohesión sirven como instrucciones de procesamiento facilitadores de la comprensión textual. Un lector hábil entonces, debe ser capaz no sólo de establecer lazos entre las distintas partes del texto, es decir establecer Cohesión textual, sino también de recuperar la información faltante sobre la base del propio conocimiento de mundo que posee. El proceso de reposición de la información implícita en el texto se realiza sobre la base de la generación de Inferencias. La capacidad de generar inferencias es fundamental para poder comprender un texto.

En función de llevar a cabo este proceso de integración activa es necesario reconocer las partes importantes del texto para no sobrecargar la memoria ni el sistema de procesamiento lingüístico con información no relevante. Es por esto, que la habilidad de reconocer la Jerarquía del texto es una capacidad esencial. Cuando el lector ha seleccionado un número suficiente de informaciones significativas, puede empezar a construir representaciones más generales del contenido del texto, es decir un "modelo mental", que irá almacenando en su memoria. La construcción de los Modelos mentales tiene la función de estructurar un cuadro de referencia general sobre la base de cinco dimensiones: espacio, tiempo, causa, motivación, protagonistas.

Es esencial, en otro plano, que los lectores posean habilidades metacognitivas generales. Por "metacognición" entendemos la capacidad que tiene un sujeto de reflexionar sobre su propio proceso de comprensión. La Intuición del texto se considera un factor metacognitivo. La identificación de tipos textuales, por ejemplo, permite generar en el lector una expectativa de lo que va a leer y orientar los objetivos de acuerdo al mismo, mejorando la comprensión del texto.

Además los lectores deben adaptar el proceso de lectura a los diferentes requerimientos y propósitos de la tarea. Un lector no lee un texto de la misma manera si su objetivo es memorizarlo, hacer un resumen del mismo o simplemente copiarlo. En cada caso, un lector hábil, empleará una estrategia diferente y es importante que el lector pueda aplicar la estrategia más adecuada a cada situación. La Flexibilidad constituye otro de los procesos metacognitivos propios del nivel textual. Por último, los lectores monitorean su propio proceso de comprensión, reconociendo los momentos en los que dicho proceso no es adecuado, mediante la detección de Errores e incongruencias.

El modelo reportado en la Figura 1 describe los componentes implicados en la comprensión textual y las relaciones que entre ellos se establecen. Se ha identificado un núcleo relativo a los componentes de contenido, un núcleo de elaboración y uno de metacognición. Estos tres núcleos convergen en la constitución de la capacidad de comprensión del texto.

\section{MÉTODO}

\section{Participantes}

Con el objetivo de lograr una muestra representativa de la población escolar de la Ciudad de Buenos Aires y Gran Buenos Aires se realizó un muestreo no aleatorio estratificado por cuotas según lugar de residencia y tipo de gestión educativa (privada o estatal). El tamaño de las cuotas se estableció siguiendo los datos censales del año 2001 proporcionados por el Instituto Nacional de Estadísticas y Censos (INDEC) respecto de la población que asiste a algún establecimiento educacional por curso escolar y sector de gestión educativa, correspondientes a la Ciudad Autónoma de Buenos Aires (CABA) y a los partidos del Gran Buenos Aires. La muestra inicial fue de 2322 alumnos que cursaban el $5^{\circ}, 6^{\circ}$ y $7^{\circ}$ grado de escuelas primarias de la Ciudad de Buenos Aires (equivalentes al $5^{\circ}$ y $6^{\circ}$ grado de escuela 
primaria y a $1^{\circ}$ año de Enseñanza Secundaria Básica (ESB) de la Provincia de Buenos Aires. (Ver Tabla 1).

Tabla 1. Muestra inicial distribuida por zona, grado y tipo de gestión educativa

\begin{tabular}{|c|c|c|c|c|c|}
\cline { 2 - 5 } \multicolumn{1}{c|}{} & $5^{\circ}$ Grado & $6^{\circ}$ Grado & $\begin{array}{c}7^{\circ} \text { Grado } \\
1^{\circ} \text { ESB }\end{array}$ & \multicolumn{2}{c|}{ Total } \\
\hline CABA & 79 & 175 & 175 & 429 \\
\hline GBA Norte & 192 & 172 & 167 & 531 \\
\hline GBA Oeste & 194 & 229 & 267 & 690 \\
\hline GBA Sur & 198 & 192 & 282 & 672 \\
\hline Total & 663 & 768 & 891 & 2322 \\
\hline & $5^{\circ}$ Grado & $6^{\circ}$ Grado & $\begin{array}{c}7^{\circ} \text { Grado } \\
1^{\circ} \text { ESB }\end{array}$ & \multicolumn{2}{c|}{ Total } \\
\hline Estatal & 428 & 283 & 472 & 1183 & $50,9 \%$ \\
\hline Privada & 235 & 485 & 419 & 1139 & $49,1 \%$ \\
\hline Total & 663 & 768 & 891 & 2322 & $100 \%$ \\
\hline
\end{tabular}

De los 2322 casos de la muestra inicial se excluyeron 230 casos: 137 fueron eliminados por no haber completado un mínimo de dos áreas debido a inasistencia y 93 por presentar uno o algunos de los siguientes criterios de exclusión: repetición de grado, antecedentes de enfermedad neurológica o psiquiátrica, trastornos del aprendizaje o trastornos sensoriales, no tener el español como lengua materna. Finalmente la muestra sobre la que se realizaron los análisis estadísticos quedó constituida por 2092 alumnos: 1037 mujeres (que representan el $49,6 \%$ de la muestra) y 1055 varones (que representan el $50,4 \%$ de la muestra). Para cada una de las áreas se obtuvo un total de entre 840 y 929 casos.

En un estudio preliminar, a partir de los primeros 300 resultados, observamos diferencias importantes entre distintas escuelas, que no estaban necesariamente relacionadas con la dependencia privada o estatal de la misma sino, con las características de su población escolar y de su organización educativa general. Se decidió entonces caracterizar las escuelas participantes en una escala de tres niveles utilizando indicadores subjetivos que permitirían categorizar las oportunidades educativas de los alumnos. Los indicadores tenidos en cuenta fueron: nivel socio-económico predominante de los alumnos asistentes, porcentaje de repetidores, ausentismo y deserción, provisión de merienda reforzada, equipamiento (biblioteca, laboratorios, sala de computación, gimnasio), jornada simple o doble, dictado de actividades extracurriculares. A partir de estos parámetros, los niveles fueron definidos de la siguiente manera:
Nivel 1: nivel socio-económico predominante bajo, merienda reforzada, alta proporción de repetidores por grado (más del $30 \%$ ), ausentismo (14\% o más) y deserción, equipamiento básico o deficiente, jornada simple, pocas o ninguna actividad extracurricular.

Nivel 2: nivel socio-económico predominante medio, baja proporción de repetidores (entre $6 \%$ y $29 \%$ ), bajo nivel de ausentismo (7\% o menos) y sin deserción, equipamiento medio (presente pero no suficiente), jornada simple o doble, actividades extracurriculares.

Nivel 3: nivel socio-económico predominante medio o alto, nula o escasa proporción de repetidores (menos del $5 \%$ ), bajo nivel de ausentismo (7\% o menos) y sin deserción, equipamiento óptimo, jornada generalmente doble, actividades extracurriculares.

Si bien esta clasificación se realizó a partir de criterios explícitos, no es exhaustiva, básicamente por dos cuestiones. En primer lugar, no agota todos los aspectos relacionados con las oportunidades educativas propias de cada sujeto (por ejemplo el nivel de escolaridad formal de los padres) ni las características de la enseñanza impartidas por la escuela. Por otro lado, resulta un indicador complejo porque combina aspectos propios del sujeto (nivel socio-económico) con otros distintivos de la institución a la que asiste (equipamiento). A pesar del cuidado que requiere el uso de este tipo de indicador, la caracterización de las escuelas en niveles permitió observar la influencia de esta variable sobre el rendimiento en comprensión lectora y obtener datos que resultan relevantes para interpretar la actuación de un individuo tanto cuando se trabaja con población escolar como con población clínica. Por otra parte, como se verá más adelante, se obtuvo una fuerte evidencia empírica sobre la influencia de los niveles en el rendimiento.

La Tabla 2 muestra la distribución de los 2092 alumnos incluidos en la muestra según el grado y las oportunidades educativas.

Tabla 2. Número de casos según grado y nivel educativo

\begin{tabular}{|c|c|c|c|c|}
\cline { 2 - 5 } \multicolumn{1}{c|}{} & $5^{\circ}$ Grado & $6^{\circ}$ Grado & $\begin{array}{c}7^{\circ} \text { Grado } \\
1^{\circ} \text { EGB }\end{array}$ & Total \\
\hline Nivel 1 & 287 & 217 & 271 & 775 \\
\hline Nivel 2 & 204 & 244 & 318 & 766 \\
\hline Nivel 3 & 108 & 234 & 209 & 551 \\
\hline Total & 599 & 695 & 798 & 2092 \\
\hline
\end{tabular}




\section{Materiales}

Leer para Comprender es un test que evalúa la comprensión de textos a partir de la valoración de 11 componentes específicos, estructurados sobre la base de 11 áreas que cuentan cada una con doce preguntas con opciones de respuesta (multiple choice). El test fue adaptado del italiano (De Beni et al., 2003) tomando en cuenta las siguientes características: se presentan textos o fragmentos que pertenecen a diversos géneros (fábula, cuento, artículos periodísticos, instrucciones, etc.) y las preguntas sobre los mismos se responden mediante una selección entre opciones múltiples. Se otorga tiempo libre para responder y el texto permanece presente (para reducir las demandas de memoria de trabajo). Las pruebas que componen el Test incluyen textos y preguntas dirigidas a evaluar componentes discretos que contribuyen a la habilidad de comprensión de textos. La especificidad de las preguntas presentadas en cada área está orientada a evaluar cada componente de manera relativamente autónoma.

\section{Procedimiento}

El Test Leer para Comprender se administró entre mayo y diciembre de 2008 en 20 escuelas. Las pruebas se aplicaron colectivamente a todos los alumnos presentes en el grado el día de la evaluación, durante una hora escolar. Se evaluaron no más de dos áreas por jornada según el grado y la dificultad de las tareas.

Para evitar interferencias con los horarios escolares y respetar las disposiciones de la dirección de la escuela, sólo en los casos en que la escuela tuviera una sola sección, se aplicó el Test completo implicando en este caso un mínimo de 6 jornadas de evaluación. Cuando la escuela contaba con más de una división por grado, la administración de las áreas se distribuyó entre las divisiones existentes.

Antes de comenzar, el evaluador verificó que los niños tuvieran al alcance los elementos necesarios y les explicó que era importante leer los textos antes de responder a las preguntar.

Se les sugirió leer detenidamente todas las opciones de respuesta y marcar la que consideraran correcta (solo una) con un círculo alrededor de la letra correspondiente. Una vez especificados estos aspectos, el evaluador entregó los protocolos del área a ser evaluada e indicó el momento en que se podía comenzar a trabajar. Se cronometró y consignó en cada protocolo el tiempo que le llevaba a cada alumno resolver el área.
Los niños podían leer a su propio ritmo los textos, las consignas y las opciones de respuesta. Cuando algún niño se mostraba nervioso o cansado se le ofrecía suspender la prueba y se consignaba este dato como comentario.

\section{Puntuación de las respuestas}

En aquellos casos en que las preguntas del test incluían opciones de respuesta, se asignaba el puntaje de la siguiente manera:

1 = elección de la respuesta correcta.

$0=$ elección de una respuesta incorrecta.

0 = elección de más de una respuesta.

$0=$ falta de respuesta.

Sin embargo, en algunos pocos ítems los alumnos debían, por ejemplo, detectar errores o reponer palabras. Para estas preguntas, se arbitraron criterios que otorgaran, también, un puntaje de 0 o 1. De esta manera, cada área podía tener un puntaje mínimo de 0 y uno máximo de 12 .

\section{RESULTADOS}

Los análisis estadísticos realizados permitieron mostrar que el Test: 1) es sensible a la progresión por grados, 2) es sensible a las diferencias de oportunidades educativas, 3) los ítems de cada una de las áreas muestran alta consistencia interna, 4) las áreas del Test correlacionan significativamente entre sí, 5) todas las áreas del Test correlacionan significativamente con una prueba externa (TECLE, Marin y Carrillo, 1999; Cuadro et al., 2009) en un análisis de validez concurrente. Sin embargo, a los fines de esta publicación, hemos decidido profundizar en los resultados del análisis de dos variables el grado y las oportunidades educativas, que resultaron altamente influyentes en el rendimiento en este Test.

\section{Efecto de las variables del sujeto: grado y nivel de oportunidades educativas}

Se estudió la influencia de dos variables del sujeto, grado y oportunidades educativas, sobre el rendimiento en las 11 áreas del Test. Se utilizaron tests no paramétricos debido a que en la mayoría de las áreas los puntajes no tenían una distribución típica.

\section{Efecto de la variable grado}

Para verificar la hipótesis de la evolución de las habilidades de comprensión de textos en relación 
al avance de la escolaridad, se analizó si existían diferencias significativas por grado en cada una de las 11 áreas del Test. Un test de Kruskal-Wallis mostró que las diferencias de puntaje entre grados eran significativas $(p<.001)$ en todas las áreas del Test, con una progresión ascendente en el puntaje entre los grados menores y los mayores. (Figura 2). La misma progresión se observó en los tiempos: a medida que el alumno avanza en su escolaridad, los tiempos utilizados disminuyen evidenciando una habilidad más desarrollada (Figura 3 ).

\section{Efecto del nivel de oportunidades educativas}

Se estudió el efecto del nivel de oportunidades educativas considerando todos los grados conjuntamente. Como se señaló anteriormente el nivel 1 , corresponde al menor nivel de oportunidades educativas y el 3 , al mayor. Una prueba de KruskalWallis mostró que las diferencias por nivel de oportunidades educativas fueron altamente significativas en las 11 áreas evaluadas por el Test $(p<.001)$.
Las diferencias son de una magnitud importante: entre los niveles extremos ( 1 y 3 ) van desde un mínimo de 1,76 puntos (área 8 ) hasta 3,90 puntos (área 6), valores considerables para una escala que va de 0 a 12 puntos.

Para concluir, es necesario aclarar que aún se están realizando otros estudios estadísticos, como por ejemplo un análisis de tendencias, que permitirá establecer si la tendencia lineal hallada hasta el momento es significativa. Los resultados serán oportunamente presentados en futuras comunicaciones.

\section{DISCUSIÓN Y CONCLUSIONES}

Más allá de los debates acerca de la complejidad del proceso de comprensión de textos, de las posibles formas de aproximación o de la especificidad o no de las dificultades, hay coincidencia en que el trastorno de la comprensión textual, con diferentes grados de severidad, es un hecho frecuente.

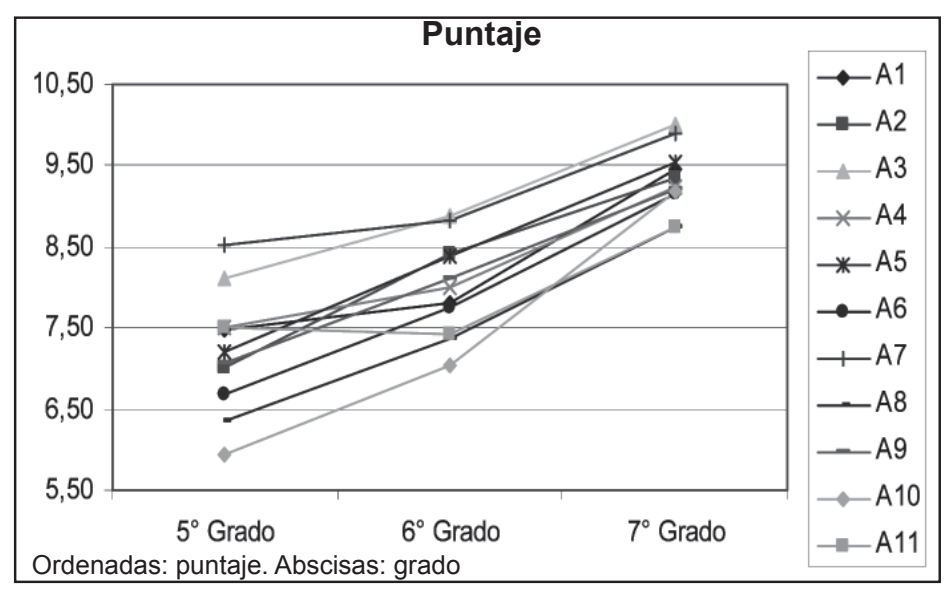

Figura 2. Gráfico de las medias de puntaje por grado

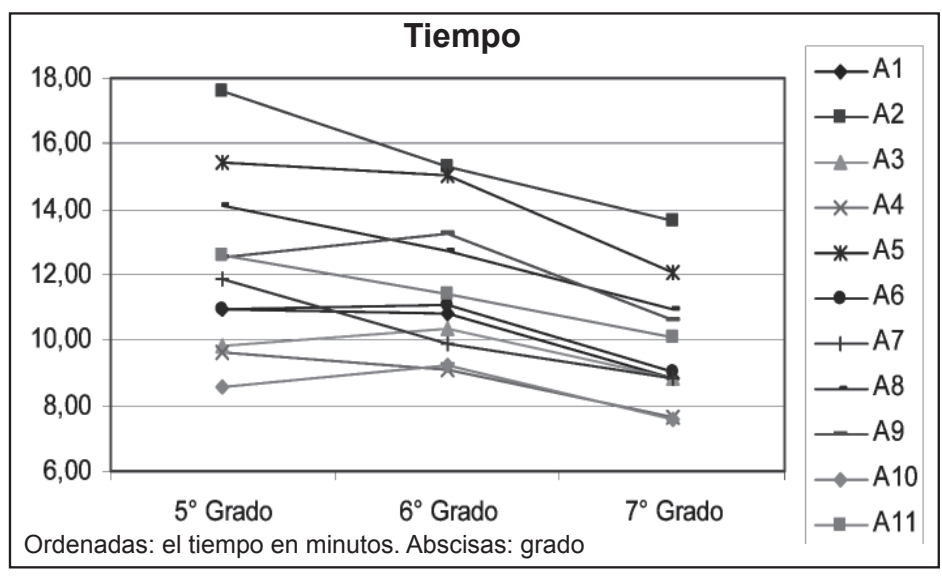

Figura 3. Gráfico de las medias de tiempo por grado 
En muchos países hay una preocupación creciente y continua por las falencias de comprensión lectora en todos los niveles de escolaridad. La preocupación surge de la percepción de los docentes y de los padres, pero las dificultades han sido efectivamente registradas por distintas investigaciones (Piacente y Tittarelli, 2006, 2008) e incluso han motivado campañas en distintos niveles gubernamentales.

El problema de las dificultades y trastornos de comprensión lectora tanto a nivel del sistema escolar como de la clínica requiere de mucha más investigación, tanto para estudiar el efecto de distintos factores causales como para medir la efectividad de las intervenciones propuestas. Pero lo que resulta evidente en este momento es que la evaluación sistemática de la comprensión de textos se ha instalado firmemente como un campo de trabajo, educativo y clínico, cuyo desarrollo resulta imprescindible para promover los avances en el tema. La evaluación no sólo es necesaria para caracterizar adecuadamente las dificultades sino también para diseñar, desarrollar y medir el efecto de intervenciones dirigidas al mejoramiento de la habilidad de comprensión lectora.

Como ya se ha anticipado, la aproximación desde un enfoque componencial constituye un aporte beneficioso para el abordaje de la comprensión textual y nuestro trabajo se ha desarrollado en esta dirección. En Argentina son pocos los instrumentos normatizados que permiten medir la comprensión textual (ver Test LEE, Defior Citoler et al., 2006) y, en función de cubrir la vacante que se genera en relación con este aspecto de la comprensión es que nos propusimos adaptar y obtener datos normativos para un instrumentos de evaluación comprensivo. En este sentido, el test Leer para Comprender resulta de utilidad para el campo de la (neuro)psicolingüística clínica y de la educación. Su particular organización lo transforma en un instrumento que permite obtener una evaluación global de un proceso complejo al tiempo que facilita la detección diferencial de problemas y la consiguiente intervención focal sobre las distintas habilidades y procesos puestos en juego.

Un análisis de los datos obtenidos permite destacar algunas cuestiones fundamentales respecto del test. En primer lugar, la aplicación de una prueba comprensiva permite obtener un perfil de rendimiento en los distintos componentes de la habilidad de comprensión lectora. Dicho perfil facilitará el planeamiento de actividades de intervención específicas. En segundo término, la sensibilidad que presenta el test respecto de las oportunidades educativas resulta fundamental para evaluar de manera real y efectiva el rendimiento escolar en comprensión de textos. Muchas dificultades en el desarrollo de las habilidades de comprensión de textos pueden estar asociadas a factores socio-económicos, culturales y/o emocionales así como a problemas propios del funcionamiento de las instituciones educativas. A pesar de que la complejidad del indicador sugiere la necesidad de utilizarlo con precaución, la caracterización de las escuelas en niveles permitió obtener datos que resultan relevantes para interpretar los resultados de un individuo tanto cuando se trabaja con población escolar como con población clínica. Si bien se necesita desarrollar mucho más la investigación en el diagnóstico y tratamiento de las dificultades y trastornos de la comprensión de textos como para adoptar un procedimiento ideal, creemos que este instrumento puede constituir un recurso de acercamiento no sólo a la detección de dificultades sino además al diseño de programas fundamentados de intervención.

\section{REFERENCIAS}

Abusamra, V., Ferreres, A., Raiter, A., De Beni. R. y Cornoldi, C. (en prensa). Leer para comprender: Test para evaluar la comprensión de textos. Buenos Aires: Editorial Paidos.

Cuadro, A., Costa, D., Trías, D. y Ponce de León, P. (2009). Manual técnico del Test de Eficacia Lectora (TECLE) de J. Marín y M. Carrillo. Montevideo: Prensa Médica Latinoamericana.

Davis (1972). Psychometric research on comprehension in reading. Reading Research Quarterly, 7, 628-678.

De Beni, R.; Cornoldi, C.; Carretti, B. y Meneghetti, B. (2003). Nuova Guida alla Comprensione del Testo. Volumen 1. Trento: Erickson.

Defior Citoler, S.; Fonseca, L.; Gottheil, B.; Aldrey A.; Jiménez Fernández, G.; Pujals, M.; Rosa, G. y Serrano Chica, F.D. (2006). LEE Test de Lectura y Escritura en Español. Buenos Aires: Paidós.

Ecco, U. (1997). Seis paseos por los bosques narrativos. Barcelona: Lumen.

Gernsbacher, M.A. (1990). Language comprehension as structure building. Hillsdale, NJ: Erlbaum.

Johnson Laird P.N. (1983). Mental models. Cambridge: Cambridge University Press.

Kintsch, W. (1988). The role of knowledge in discourse comprehension: A construction-integration model. Psychological Review, 95 (2), 163-182.

Marin, J. y Carrillo, M. (1999) Test Colectivo de Eficacia Lectora (TECLE). Manuscrito no publicado. Departamento de Psicología Básica y Metodología. Universidad de Murcia.

Piacente, T. \& Tittarelli, A. M. (2008). Comprensión y producción de textos en alumnos universitarios. La reformulación textual. Orientación y Sociedad. V. 7, 125-134. ISNN 1515-6877.

van Dijk T. y Kintsch W. (1983). Strategies of discourse comprehension. New York: Academic Press. 